\title{
Hereditary cancer testing in patients with ovarian cancer using a 25-gene panel
}

\author{
Lucy R Langer, MD, MSHS; ${ }^{a}$ Heidi McCoy, MS, CGC; John Kidd, MS; ; Brent Evans, MS; \\ Krystal L Brown, PhD; Jennifer Saam, MS, CGC, PhD; ${ }^{\text {b }}$ and Richard Wenstrup, MD
}

${ }^{\mathrm{a}}$ Compass Oncology, Portland, Oregon, and ${ }^{\mathrm{b}}$ Myriad Genetic Laboratories Inc, Salt Lake City, Utah

Background The identification of pathogenic mutations in genes that increase the risk of ovarian cancer has an impact on the clinical management of patients, including decisions about surveillance, chemoprevention, and risk-reducing surgical interventions. Mutations in hereditary cancer susceptibility genes account for up to $20 \%$ of ovarian cancers in the US.

Objectives To analyze the mutations detected using a multigene panel in patients with a personal history of ovarian cancer and evaluate the emerging use of panel testing.

Methods We identified 3,088 patients with ovarian cancer whose samples had been submitted to a large commercial laboratory for genetic testing with a 25 -gene hereditary-risk panel. The frequency and spectrum of mutations were analyzed according to clinical factors (ancestry, age at testing, testing criteria).

Results Deleterious or suspected deleterious mutations were identified in 419 patients (13.6\%), 7 of whom had mutations in 2 different genes. Testing patients using the 25 -gene panel increased the number of positive test results in ovarian cancer patients by $53.8 \%$ over $B R C A 1 / 2$ testing alone, showing the benefit of using a panel approach in this population. In all, $27.2 \%$ of patients with positive test results had mutations that would not have been identified by single-syndrome genetic testing for hereditary breast and ovarian cancer or Lynch syndrome.

Limitations Clinical histories collected by test request form; retrospective study.

Conclusions Our results demonstrate the benefits of multigene panels for patients with personal history of ovarian cancer, particularly for the identification of moderate-penetrance mutations that would not otherwise be identified by single-syndrome testing. Funding Myriad Genetic Laboratories

G enetic testing offers the opportunity to identify patients with an elevated risk for hereditary cancers and allows appropriate changes in medical management for these patients and their family members. Early detection of ovarian cancer is difficult owing to the anatomical location in the abdomen and growth pattern of the tumors. As a result, the majority of ovarian cancer patients are diagnosed at an advanced stage. ${ }^{1}$ Genetic screening is of particular importance for cancers that are difficult to diagnose or that have historically poor prognoses, because it provides family members who may inherit the increased genetic risk the opportunity to use prevention options such as surgery or chemoprevention that might not be appropriate for the general-risk population. There is a strong hereditary risk of ovarian cancer, as women who have a first-degree relative with ovarian cancer have a twoto six-fold higher risk of developing the cancer themselves. ${ }^{2}$ Furthermore, mutations in hereditary cancer susceptibility genes account for $11 \%-15 \%$ of cases of epithelial ovarian cancer ${ }^{3,4}$ and up to $20 \%$ of all ovarian cancers, ${ }^{5}$ with hereditary breast and ovarian cancer (HBOC) and Lynch syndrome comprising the majority of these cases. ${ }^{5,6}$

Traditional single-syndrome genetic testing is reliant on clinical suspicion of a particular cancer syndrome susceptibility based on overt personal and/or family history. Today, all patients with epithelial ovarian cancer meet National Comprehensive Cancer Network (NCCN) guidelines for $B R C A 1$ and $B R C A 2$ genetic testing. ${ }^{7}$ Women with $B R C A 1$ and $B R C A 2$ mutations have a $23 \%-44 \%$ risk of developing ovarian cancer ${ }^{8-10}$ and a $6.8 \%-12.7 \%$ risk of developing ovarian cancer within 10 years of a breast cancer diagnosis. ${ }^{11}$ As a result, it is recommended that women with a $B R C A 1$ or $B R C A 2$ mutation have a bilateral salpingo-oophorectomy after child bearing is complete. ${ }^{12}$ Thus, screening and early identification of hereditary ovarian cancer is key for successful clinical intervention.

Although most health care providers associate ovarian cancer with only HBOC, the lifetime risk of ovarian cancer for women with Lynch syndrome

Accepted for publication October 5, 2015. Correspondence: Lucy R Langer, MD, MSHS; Lucy.Langer@USOncology com. Disclosures: Dr Langer is a paid consultant with Myriad. Ms McCoy, Mr Kidd, Mr Evans, Dr Brown, Dr Samm, and Dr Wenstrup are employees of Myriad and receive salary and stock options as compensation. JCSO 2016;14:314-319. (C2016 Frontline Medical Communications. doi: 10.12788/jicso.0181. 
is $8 \%$, compared with $1.4 \%$ in the general population. ${ }^{3,13}$ This is coupled with an earlier age at onset, with Lynch syndrome patients being diagnosed between the ages of 42-49 years, compared with 60-65 years for sporadic ovarian cancer. ${ }^{14-16}$ Accordingly, patients with ovarian cancer and personal or family history of colon and/or endometrial cancer may meet guidelines for Lynch syndrome testing. Mutations in BRIP1, ${ }^{17}$ TP53, ${ }^{18}$ STK11 ${ }^{19}$ RAD $51 C,{ }^{20,21}$ and $R A D 51 D^{22}$ have also been identified as carrying an increased ovarian cancer risk.

In response to this type of genetic heterogeneity in many hereditary cancers, academic and commercial laboratories have introduced next-generation sequencing (NGS) platforms that simultaneously assay multiple genes associated with a spectrum of hereditary genetic disorders. This is typically a combination of well-characterized, highly penetrant genes associated with individual hereditary cancer syndromes, such as HBOC and Lynch syndrome, as well as other genes with known cancer risks. These panels allow clinicians to assay sequence data from many genes concurrently, often at a fraction of the cost of traditional methods. ${ }^{23-26}$ This offers a method to overcome the genetic and phenotypic heterogeneity of genetic disorders by allowing patients to circumvent the lengthy diagnostic algorithm associated with sequential single-gene genetic testing. ${ }^{27}$ In addition, NGS platforms have increased the appreciation of the role of mutations in more recently discovered genes in hereditary cancer susceptibility.

The aim of the study presented here was to examine the advantages and limitations of using multigene panels in patients with a personal history of ovarian cancer. To do this, we performed a retrospective database analysis of patients with personal history of ovarian cancer whose samples had been submitted to a large commercial laboratory for hereditary cancer genetic testing.

\section{Methods}

We performed a retrospective database analysis to identify all patients with a personal history of ovarian cancer who underwent genetic testing with a 25-gene hereditary cancer panel from September 4, 2013-November 17,2014 . This study included all ovarian cancers, including fallopian tube, peritoneal, and nonepithelial ovarian cancer. All patient data regarding clinical history was obtained from health care provider reports on test requisition forms.
All of the patients received genetic testing using the 25-gene panel, which includes $B R C A 1, B R C A 2$, the mismatch-repair genes (MLH1, MSH2, MSH6, PMS2), APC, MUTYH, CDKN2A, CDK4, PALB2, CHEK2, SMAD4, BMPR1A, STK11, TP53, CDH1, PTEN, ATM, NBN, BARD1, BRIP1, RAD51C, and RAD51D. Sequencing and large-rearrangement analysis was performed for all genes on the panel except for $E P C A M$, for which only large rearrangement analysis was performed. This next-generation hereditary cancer panel has been validated by Sanger sequencing, the details of which have been previously described. ${ }^{28}$

A descriptive analysis was performed to describe the frequency and distribution of mutations identified with the 25-gene panel among women with a personal history of ovarian cancer. Additional analyses were performed based on self-identified ancestry, age at testing, and whether tested individuals met NCCN testing criteria based on personal and family cancer history. The $2013 \mathrm{NCCN}$ guidelines for HBOC testing were applied, excluding the contribution from prostate cancer as Gleason score is not documented on the test requisition form. ${ }^{7}$ Women with a first- or second-degree relative who met revised Bethesda criteria ${ }^{29}$ or had a diagnosis of endometrial cancer before the age of 50 were defined as meeting Lynch syndrome testing criteria. The proportion of mutations identified with panel testing that would not have been identified with single-syndrome testing was evaluated based on the mutations identified

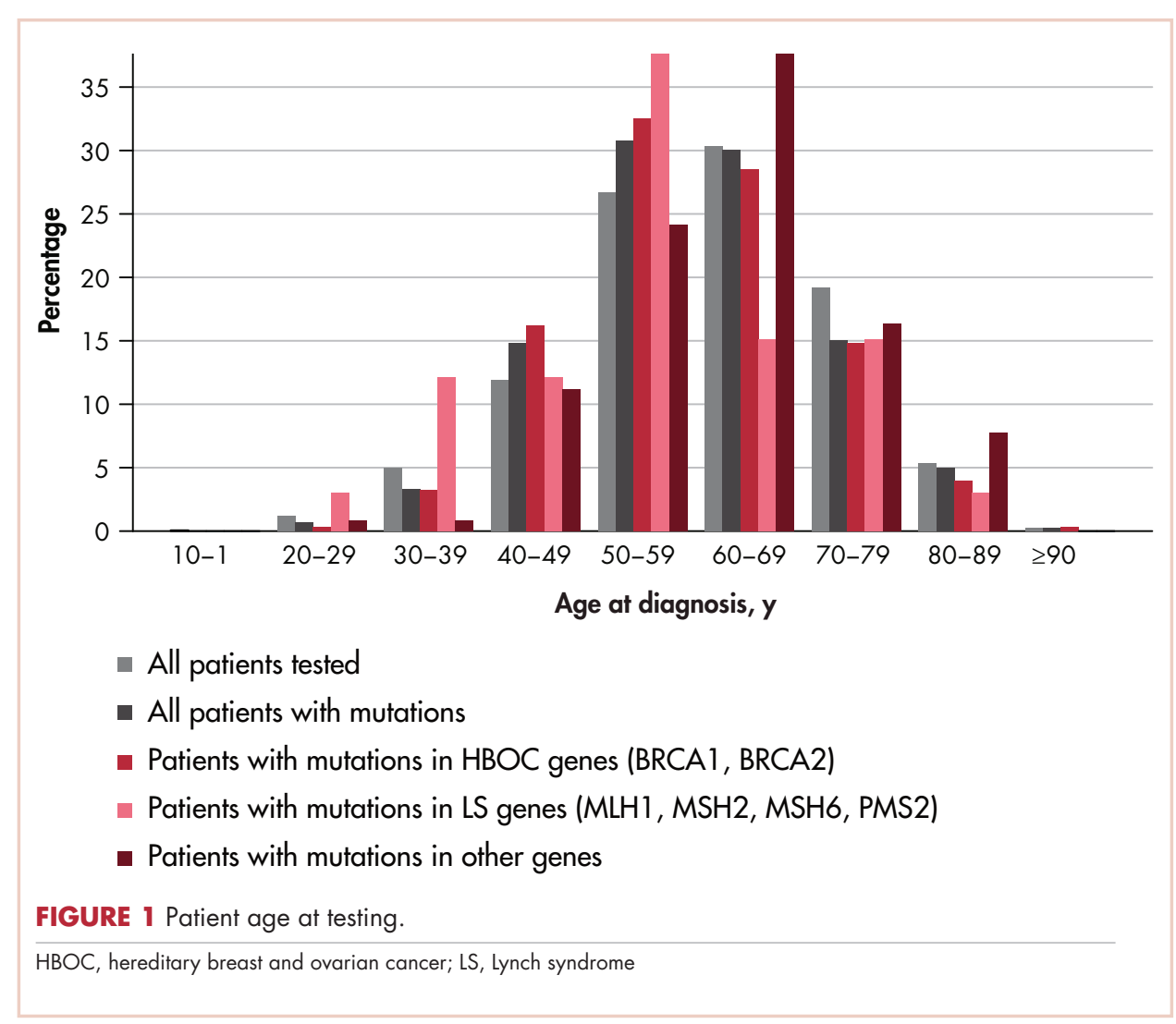


using the panel that occurred in genes included in HBOC testing (BRCA1 and BRCA2) or Lynch syndrome testing (MLH1, MSH2, MSH6, PMS2, EPCAM).

\section{Results}

\section{Patient demographics}

During the study, 3,088 patients with a personal history of ovarian cancer underwent genetic testing using the 25 -gene panel. Physicians had the option of explicitly specifying nonepithelial ovarian cancer on the test request forms as well as writing in specific cancer types of the peritoneum and/or fallopian tube. Among the patients reported, 2,868 (92.9\%) were diagnosed with ovarian cancer. The remaining patients were diagnosed with cancer of the peritoneum (2.3\%), fallopian tube $(2.1 \%)$, ovary (nonepithelial; $1.9 \%)$, ovary and fallopian tube $(0.5 \%)$, or ovary and peritoneum $(0.3 \%)$.

The majority of patients who were tested $(57.1 \%)$ were diagnosed between the ages of 50 and 69 years (Figure 1). Specifically, 824 (26.7\%) were diagnosed between ages 50 and 59 years, and 938 (30.4\%) were diagnosed between ages 60 and 69 years. The majority of patients $(60.3 \%)$ were of European ancestry, with the remaining patients being of Latin American/Caribbean (5.8\%), African (3.6\%), Asian (3.1\%), Ashkenazi Jewish (2.3\%), Near/Middle Eastern (0.7\%), and Native American (0.6\%) descent (Table 1). In addition, $10.9 \%$ of patients specified multiple ancestries, and $12.7 \%$ did not specify any ancestry.

\section{Mutation prevalence}

Deleterious or suspected deleterious mutations were identified in 419/3,088 (13.6\%) patients. Of those mutations, $65.0 \%$ (277) were identified in BRCA1/BRCA2 (Table 2). Mutations in Lynch syndrome-associated genes (MLH1, MSH2, MSH6, PMS2) accounted for 33 (7.8\%) of the pathogenic mutations. The remaining 116 (27.2\%) of mutations were identified in genes that are not associ-

TABLE 1 Ethnicity of patients with a personal history of ovarian cancer

\begin{tabular}{|lccc|}
\multicolumn{1}{|c}{ Ancestry } & $\begin{array}{c}\text { Total patients, } \\
\mathbf{n}(\%)\end{array}$ & $\begin{array}{c}\text { Positive patients, } \\
\text { n (\%) }\end{array}$ & $\begin{array}{c}\text { Positive } \\
\text { rate, \% }\end{array}$ \\
\hline African & $110(3.6)$ & $13(3.1)$ & 11.8 \\
\hline Ashkenazi & $70(2.3)$ & $8(1.9)$ & 11.4 \\
\hline Asian & $97(3.1)$ & $15(3.6)$ & 15.5 \\
\hline $\begin{array}{l}\text { European } \\
\text { Latin American/ } \\
\text { Caribbean }\end{array}$ & $1863(60.3)$ & $239(57.0)$ & 12.8 \\
\hline $\begin{array}{l}\text { Native American } \\
\text { Near Eastern/ } \\
\text { Middle Eastern }\end{array}$ & $18(0.6)$ & $35(8.4)$ & 19.7 \\
\hline $\begin{array}{l}\text { Multiple } \\
\text { None specified }\end{array}$ & $337(10.9)$ & $23(1.2)$ & 27.8 \\
\hline
\end{tabular}

ated with $\mathrm{HBOC}$ or Lynch syndrome. A portion of those mutations were found in BRIP1 (4.9\%), RAD51C (4.5\%), RAD51D (0.9\%), and TP53 (0.5\%). Additional mutations were identified in ATM, CHEK2, PALB2, NBN, BARD1, $A P C, P T E N$, and $C D K N 2 A$. Seven patients were identified as having 2 pathogenic mutations. This included 4 patients with a $B R C A 1$ mutation who had a second mutation in either ATM (2), BARD1 (1), or PMS2 (1). There were also 3 patients with a $B R C A 2$ mutation who had a second mutation in either $A T M(1), N B N(1)$, or PALB2 (1). One or more variant of uncertain significance (VUS) was identified in $36.9 \%$ of patients (Figure 2).

The distribution of ancestries among positive patients was similar to that of all tested patients (Table 1). Most ancestries had positive mutation rates ranging from $11.4 \%-19.7 \%$. Patients of Native American and Near/Middle Eastern descent fell outside of this range; however, both of these ancestries had 5 or fewer patients with a pathogenic mutation.

\section{NCCN testing criteria}

In all, 2,410 patients (78.4\%) tested met NCCN guidelines for HBOC testing only, $10(0.3 \%)$ met guidelines for Lynch syndrome testing only, and 630 (20.5\%) met NCCN

TABLE 2 Mutations among patients with a personal history of ovarian cancer

\begin{tabular}{|c|c|}
\hline Gene & Frequency (\%) \\
\hline \multicolumn{2}{|l|}{$\mathrm{HBOC}$} \\
\hline$B R C A I$ & $153(35.9)$ \\
\hline BRCA2 & $124(29.1)$ \\
\hline \multicolumn{2}{|c|}{ Lynch syndrome } \\
\hline PMS2 & $12(2.8)$ \\
\hline MSH6 & $11(2.6)$ \\
\hline MLHI & 5 (1.2) \\
\hline $\mathrm{MSH} 2$ & $5(1.2)$ \\
\hline \multicolumn{2}{|l|}{ Other } \\
\hline ATM & $24(5.6)$ \\
\hline$B R I P 1$ & $21(4.9)$ \\
\hline CHEK2 & $19(4.5)$ \\
\hline RAD51C & $19(4.5)$ \\
\hline PALB2 & $13(3.1)$ \\
\hline NBN & $6(1.4)$ \\
\hline BARDI & $4(0.9)$ \\
\hline RAD51D & $4(0.9)$ \\
\hline$A P C$ & $2(0.5)$ \\
\hline TP53 & $2(0.5)$ \\
\hline CDKN2A & $1(0.2)$ \\
\hline PTEN & $1(0.2)$ \\
\hline
\end{tabular}




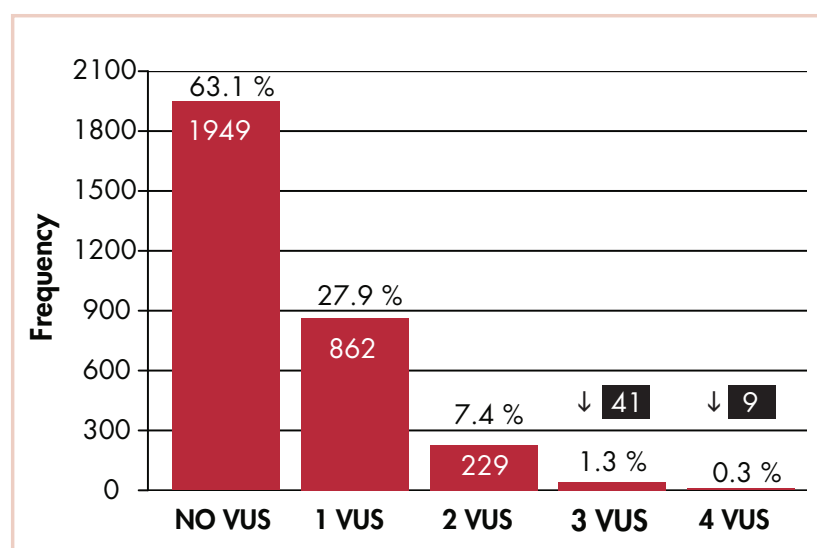

FIGURE 2 Variants of uncertain significance in tested patients.

VUS, variants of uncertain significance

guidelines for both syndromes (Figure 3). A small fraction of patients $(0.8 \%)$ did not meet either NCCN testing criteria. In addition, nearly all patients with a pathogenic mutation met NCCN testing criteria, with the exception of 2 patients (Figure 3). Specifically, 337 (81.2\%) of all mutation-positive patients met the guidelines for HBOC only, and 76 (18.3\%) met guidelines for both HBOC and Lynch syndrome. Nearly all patients with a $B R C A 1$ or $B R C A 2$ mutation met NCCN testing criteria for $\mathrm{HBOC}$, with $86.1 \%$ meeting criteria for HBOC only and $13.6 \%$ meeting criteria for both HBOC and Lynch syndrome (Figure 3). This shows a strong correlation between $\mathrm{HBOC}$-associated mutations and the corresponding NCCN guidelines.

Among patients with Lynch syndrome-associated mutations, $42.4 \%$ met NCCN criteria for HBOC but not Lynch syndrome, and $57.6 \%$ met NCCN guidelines for both HBOC and Lynch syndrome (Figure 3). However, 26.8\% of mutations were identified in genes not associated with HBOC or Lynch syndrome, suggesting that although current testing guidelines identified nearly all patients with a hereditary cause for ovarian cancer in this study, more than a quarter of the pathogenic mutations identified would have been missed with single-syndrome testing alone.

\section{Age at diagnosis}

Similar to the trend observed for patients tested, 255 patients $(60.9 \%)$ with a mutation were diagnosed between the ages of 50 and 69 . The subset of patients with $B R C A 1$ or $B R C A 2$ mutations follow the same age distribution, with 169 (61.0\%) being diagnosed between the ages of 50 and 69 and an overall age distribution similar to all mutation-positive patients (Figure 1). This distribution is slightly shifted in patients with mutations in Lynch syndrome-associated genes and genes not associated with either HBOC or Lynch syndrome (Figure 1). Among patients with Lynch syndrome mutations, 13 (39.4\%) were diagnosed between the ages of 50 and 59 , and $5(15.2 \%)$ were diagnosed between 60 and
69. Care should be taken in the interpretation of these data, because only 33 total patients were identified with Lynch syndrome-associated mutations. Among patients with mutations in genes not associated with HBOC or Lynch syndrome, $24.1 \%$ were diagnosed between ages 50 and 59 , whereas $38.8 \%$ were diagnosed between 60 and 69 years of age, showing a slight shift to the older age group.

\section{Discussion}

Our results support recent studies that demonstrate the potential benefit of multigene panel testing for cancer susceptibility genes in individuals with personal and/or family history suggestive of a hereditary cancer syndrome. ${ }^{5,28,30}$ This includes a study by Walsh and colleagues that used a 21-gene panel that included many of the genes investigated here and reported a $23 \%$ positive mutation rate among patients with ovarian cancer. ${ }^{5}$ Of the 3,088 patients with personal history of ovarian cancer who underwent genetic testing with the 25-gene panel, 419 (13.6\%) had a deleterious or suspected deleterious mutation. Although that is lower than the mutation rate reported by Walsh and colleagues, the present study investigated a significantly larger and broader patient population in an effort to more accurately represent hereditary cancer risk.

Testing patients using the 25 -gene hereditary cancer panel increased the number of positive test results in ovarian cancer patients by $53.8 \%$ over BRCA1 and BRCA2 testing alone. This is in agreement with the study findings of Walsh and colleagues, which showed a $35 \%$ increase in mutations detected over BRCA1 and BRCA2 testing alone. ${ }^{5}$ The increased mutation prevalence observed in the present study is likely reflective of the broad patient population and the number of genes included here.

The majority of patients who were tested met NCCN testing criteria for HBOC and/or Lynch syndrome. The small fraction of patients that did not meet any NCCN testing criteria reflects the inclusion of nonepithelial ovarian cancer in patient selection, which is not part of NCCN testing criteria. The fraction of patients who didn't meet any guidelines $(0.8 \%)$ is lower than the fraction of patients with a personal history of nonepithelial ovarian cancer (1.9\%). This suggests that the family histories of most of these patients are sufficient for them to be included in current testing criteria.

All patients with a pathogenic mutation met NCCN guidelines for either HBOC or Lynch syndrome, with the exception of 2 patients with non-epithelial ovarian cancer. One patient who did not meet any guidelines was found to have a mutation in BRCA1; however, the patient's father (diagnosed with pancreatic cancer, age 53) would have met HBOC testing guidelines based on a cousin with ovarian cancer (diagnosed at age 62) and a cousin with breast cancer (diagnosed at age 47). Although these family members were too far removed for the patient to meet guidelines herself, the $B R C A 1$ mutation identified is not altogether surprising. The other patient who did not meet either HBOC 


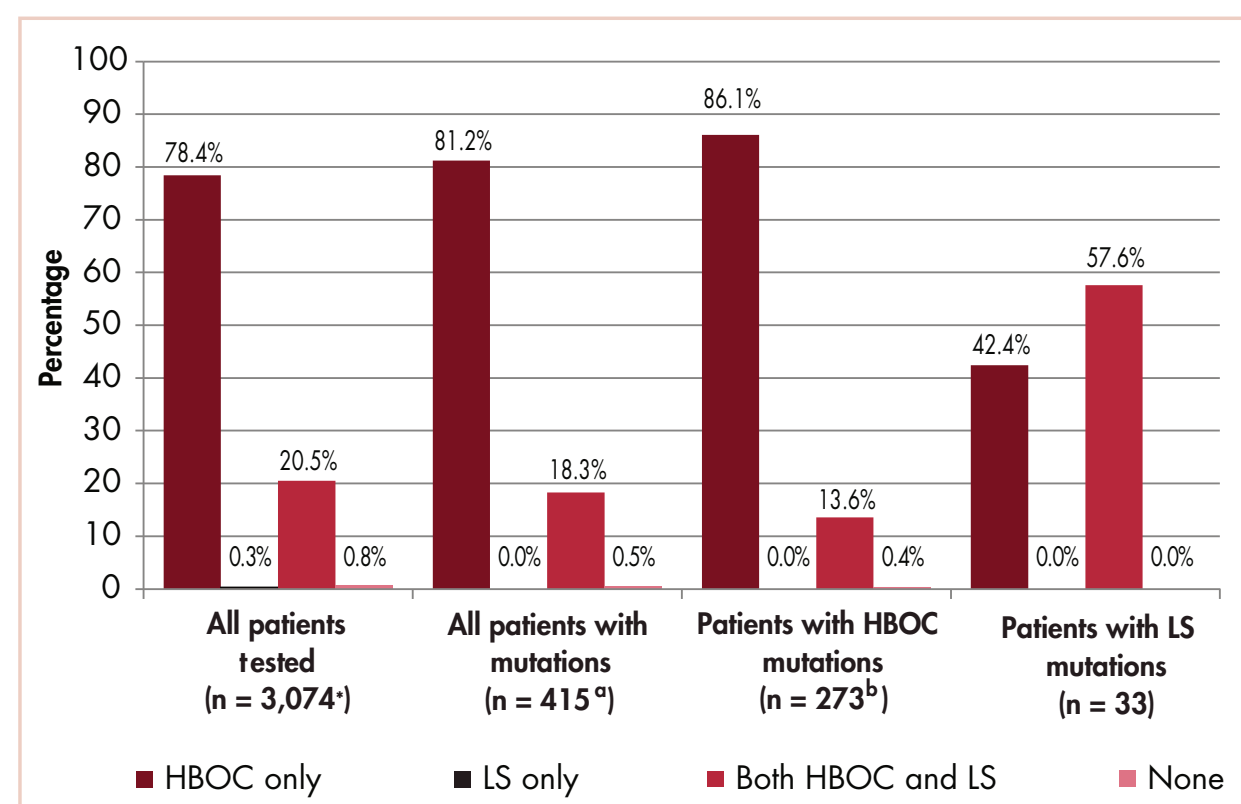

FIGURE 3 Adherence to National Comprehensive Cancer Network testing criteria.

HBOC, hereditary breast and ovarian cancer; LS, Lynch syndrome; NCCN, National Comprehensive Cancer Network

aExcludes 14 patients who were missing NCCN guideline information. ${ }^{b}$ Excludes 4 patients who were missing NCCN guideline information.

tified in BRIP1, RAD51C, RAD51D, and TP53, respectively. The largest fraction of mutations in genes not associated with $\mathrm{HBOC}$ or Lynch syndrome was identified in ATM (5.6\%), which suggests a possible increased risk for ovarian cancer, which should be followed up in future studies.

The nature of panel testing can result in the identification of some incidental findings. Two patients were identified as having a mutation in $A P C$, which is not associated with ovarian cancer. The family histories for both patients included multiple primary family members diagnosed with colon cancer before age 50 . Although it is likely that the ovarian cancer in these patients was incidental, the mutations in $A P C$ are consistent with their family histories and highlights the benefit of panel testing in which genetic causes can be assessed for multiple syndromes. The proportion of patients with a VUS is also not surprising given the early development of this 25-gene panel test.

or Lynch syndrome testing guidelines had a mutation in PTEN. The patient's family history included a brother with kidney cancer (diagnosed at age 38), a mother with skin cancer (diagnosed at age 70, melanoma not specified), and a maternal grandmother with lung cancer (age at diagnosis not specified). While it is not a classic presentation, it shares features of Cowden syndrome.

There are minimal differences between the overall age at diagnosis distribution for all patients tested and patients with a mutation (Figure 1). This suggests that age is not a significant indicator of hereditary risk for ovarian cancer, which is consistent with previous studies. ${ }^{5}$ Despite some small differences in patients with mutations in HBOC- compared with Lynch syndrome-associated genes, the overall age distribution of patients with a mutation in all gene groups examined here were similar, with about $60 \%$ of patients being diagnosed between the ages of 50 and 69 years. That shows that age at diagnosis for patients with ovarian cancer is not a significant predictor of the rate of positive mutation for any of the individual gene groups investigated here (HBOCassociated, Lynch syndrome-associated, all others).

A substantial proportion of patients (27.2\%) with a mutation had mutations that would not have been identified by single-syndrome genetic testing for either HBOC or Lynch syndrome. Findings from previous studies have shown that mutations in genes such as BRIP $1{ }^{17}$ RAD $51 C,{ }^{20,21}$ $R A D 51 D,^{22}$ and TP5 $3^{18}$ have an increased risk for ovarian cancer. This was also observed in the present study, with $4.9 \%$, $4.5 \%, 0.9 \%$, and $0.5 \%$ of patients with mutations being iden-
This is commensurate with the proportion of VUSs identified by single-gene assays early in their development. ${ }^{31,32} \mathrm{It}$ is anticipated that this number will decrease as a result of both the impact of targeted efforts directed at determining the pathogenicity of variants and increasing availability of data as more patients are tested. ${ }^{31,32}$

The largest proportion of mutations identified here (65.0\%) was in HBOC-associated genes (BRCA1 and BRCA2). Nearly all patients with an HBOC mutation met NCCN guidelines for either HBOC or both HBOC and Lynch syndrome. All of the patients identified as having 2 mutations had one mutation in either BRCA1 or BRCA2. Had these patients undergone single-syndrome testing, it is unlikely that a health care provider would have followed a positive HBOC result with additional testing. However, the use of the 25 -gene panel here identified these additional mutations to allow more appropriate medical management decisions to be made for these patients and their family members.

Although ovarian cancer is most commonly associated with HBOC, the data presented here show that $7.8 \%$ of the pathogenic mutations identified were in Lynch syndrome-associated genes (Table 2). Despite the increased ovarian cancer risk, many patients who are at risk for Lynch syndrome are tested for HBOC only. This results from the lack of clinical recognition of Lynch syndrome. For example, a patient who met HBOC and Lynch syndrome guidelines based on a mother with uterine cancer (diagnosed at age 48) and a maternal grandmother with ovarian cancer (diagnosed at age 74) was found to have a mutation in 
MSH2. Another patient met guidelines for both HBOC and Lynch syndrome based on a father with colon cancer (diagnosed at age 86) and multiple paternal ovarian and breast cancers. This patient was found to have a mutation in both $B R C A 1$ and $P M S 2$. In both cases, it is unlikely that the physician would have followed either the negative or positive single-syndrome HBOC test results with Lynch syndrome testing to identify these pathogenic mutations.

In addition, Lynch syndrome guidelines may not capture all patients at risk for Lynch syndrome. In this study, $42.4 \%$ of ovarian cancer patients who had Lynch syndrome mutations did not meet Lynch syndrome guidelines (Figure 3). For example, a patient who met HBOC guidelines and had a family history of breast cancer (maternal aunt, diagnosed at age 66; maternal great aunt, diagnosed at age 50) was found to have a mutation in MSH6. This clinically actionable mutation

\section{References}

1. Crispens MA. Endometrial and ovarian cancer in lynch syndrome. Clin Colon Rectal Surg. 2012;25:97-102.

2. Neesham D. Ovarian cancer screening. Aust Fam Physician. 2007;36:126-128.

3. Aarnio M, Sankila R, Pukkala E, et al. Cancer risk in mutation carriers of DNA-mismatch-repair genes. Int J Cancer. 1999;81:214-218.

4. Bewtra C, Watson P, Conway T, Read-Hippee C, Lynch HT. Hereditary ovarian cancer: a clinicopathological study. Int J Gynecol Pathol. 1992;11:180-187.

5. Walsh T, Casadei S, Lee MK, et al. Mutations in 12 genes for inherited ovarian, fallopian tube, and peritoneal carcinoma identified by massively parallel sequencing. Proc Natl Acad Sci USA. 2011;108:18032-18037.

6. Lu K, ed Clinical relevance of hereditary endometrial cancer In: Hereditary gynecologic cancer: risk, prevention, and management. 1st ed. New York, NY: Informa Healthcare; 2008.

7. Daly M, Pilarski R, Axilbund JE, et al. Genetic/familial high-risk assessment: breast and ovarian. Version 1.2013. NCCN Clinical Practice Guidelines in Oncology. 2013. http://www.nccn.org/professionals/physician_gls/pdf/genetics_screening.pdf. Accessed April 26, 2016.

8. Easton DF, Ford D, Bishop DT. Breast and ovarian cancer incidence in BRCA1-mutation carriers. Breast Cancer Linkage Consortium. Am J Hum Genet. 1995;56:265-271.

9. Ford D, Easton DF, Bishop DT, Narod SA, Goldgar DE. Risks of cancer in BRCA1-mutation carriers. Breast Cancer Linkage Consortium. Lancet. 1994;343:692-695.

10. Ford D, Easton DF, Stratton M, et al. Genetic heterogeneity and penetrance analysis of the BRCA1 and BRCA2 genes in breast cancer families. The Breast Cancer Linkage Consortium. Am J Hum Genet. 1998;62:676-689.

11. Metcalfe KA, Lynch HT, Ghadirian P, et al. The risk of ovarian cancer after breast cancer in BRCA1 and BRCA2 carriers. Gynecol Oncol. 2005;96:222-226.

12. Morgan RJ, Jr., Alvarez RD, Armstrong DK, et al. Ovarian cancer, version 2.2013. J Natl Compr Canc Netw. 2013;11:1199-1209.

13. Watson P, Vasen HF, Mecklin JP, et al. The risk of extra-colonic, extra-endometrial cancer in the Lynch syndrome. Int J Cancer. 2008;123:444-449.

14. Engel C, Loeffler M, Steinke V, et al. Risks of less common cancers in proven mutation carriers with lynch syndrome. J Clin Oncol. 2012;30:4409-4415.

15. Ketabi Z, Bartuma K, Bernstein I, et al. Ovarian cancer linked to Lynch syndrome typically presents as early-onset, non-serous epithelial tumors. Gynecol Oncol. 2011;121:462-465.

16. Watson P, Butzow R, Lynch HT, et al. The clinical features of ovarian cancer in hereditary nonpolyposis colorectal cancer. Gynecol Oncol. 2001;82:223-228. would likely have been missed with single-syndrome testing.

The results presented here demonstrate the potential benefits of multigene panels in patients with personal history of ovarian cancer. Although HBOC is strongly associated with ovarian cancer, $35 \%$ of the pathogenic mutations identified here occurred in genes other than $B R C A 1$ and $B R C A 2$. Although current NCCN guidelines identified the majority of patients with a mutation in this analysis, these additional genes would not have been identified by single-syndrome testing. This suggests that multigene panels may offer the opportunity to provide better patient care for both affected patients and unaffected family members.

\section{Acknowledgment}

The authors acknowledge the efforts of the clinicians and patients who have made this work possible.

17. Rafnar T, Gudbjartsson DF, Sulem P, et al. Mutations in BRIP1 confer high risk of ovarian cancer. Nat Genet. 2011;43:1104-1107.

18. Schneider K, Zelley K, Nichols KE, Garber J. Li-Fraumeni Syndrome. In: Pagon RA, Adam MP, Ardinger HH, et al., eds. GeneReviews(R). Seattle, Washingto: University of Washington, Seattle; 1993.

19. Burt RW, Barthel JS, Dunn KB, et al. NCCN clinical practice guidelines in oncology. Colorectal cancer screening. J Natl Compr Canc Netw. 2010;8:8-61.

20. Coulet F, Fajac A, Colas C, et al. Germline RAD51C mutations in ovarian cancer susceptibility. Clin Genet. 2013;83:332-336.

21. Loveday C, Turnbull C, Ruark E, et al. Germline RAD51C mutations confer susceptibility to ovarian cancer. Nat Genet. 2012;44:475-476; author reply 476.

22. Loveday C, Turnbull C, Ramsay E, et al. Germline mutations in RAD51D confer susceptibility to ovarian cancer. Nat Genet. 2011;43:879-882.

23. Guttmacher AE, McGuire AL, Ponder B, Stefansson K. Personalized genomic information: preparing for the future of genetic medicine. Nat Rev Genet. 2010;11:161-165.

24. Rehm HL. Disease-targeted sequencing: a cornerstone in the clinic. Nat Rev Genet. 2013;14:295-300.

24. Roberts NJ, Vogelstein JT, Parmigiani G, Kinzler KW, Vogelstein B, Velculescu VE. The predictive capacity of personal genome sequencing. Sci Transl Med. 2012;4:133ra158.

26. Walsh T, Lee MK, Casadei S, et al. Detection of inherited mutations for breast and ovarian cancer using genomic capture and massively parallel sequencing. Proc Natl Acad Sci USA. 2010;107:12629-12633.

27. Selkirk CG, Vogel KJ, Newlin AC, et al. Cancer genetic testing panels for inherited cancer susceptibility: the clinical experience of a large adult genetics practice. Fam Cancer. 2014.

28. Tung N, Battelli C, Allen B, et al. Frequency of mutations in individuals with breast cancer referred for BRCA1 and BRCA2 testing using next-generation sequencing with a 25-gene panel. Cancer. 2014.

29. Umar A, Boland CR, Terdiman JP, et al. Revised Bethesda Guidelines for hereditary nonpolyposis colorectal cancer (Lynch syndrome) and microsatellite instability. J Natl Cancer Inst. 2004;96(4):261-268.

30. American Cancer Society. Ovarian Cancer: 2014. http://www.cancer. org/cancer/ovariancancer/detailedguide/. Last revised February 4, 2016. Accessed February 9, 2015.

31. Kurian AW, Hare EE, Mills MA, et al. Clinical evaluation of a multiple-gene sequencing panel for hereditary cancer risk assessment. J Clin Oncol. 2014;32:2001-2009.

32. Eggington JM, Bowles KR, Moyes K, et al. A comprehensive laboratory-based program for classification of variants of uncertain significance in hereditary cancer genes. Clin Genet. 2014;86:229-237. 